\title{
Influência do boro, de fontes de nitrogênio e do pH do solo no controle de hérnia das crucíferas causada por Plasmodiophora brassicae
}

\author{
Lucimeris Ruaro ${ }^{1}$, Vismar da C. Lima Neto ${ }^{1} \&$ Paulo Justiniano Ribeiro Júnior ${ }^{2}$ \\ ${ }^{1}$ Departamento de Fitotecnia e Fitossanitarismo; ${ }^{2}$ Departamento de Estatística, Universidade Federal do Paraná 80035-050, \\ Curitiba, PR, Brasil
}

Autor para correspondência: Lucimeris Ruaro,e-mail: lucimeris@ufpr.br

\begin{abstract}
RESUMO
$\mathrm{O}$ efeito de doses de boro (B) e de fontes de nitrogênio $(\mathrm{N})$ em diferentes níveis de $\mathrm{pH}$ do solo no controle de Plasmodiophora brassicae, agente causal da hérnia das crucíferas foi avaliado, em solo artificialmente infestado com o patógeno, imediatamente após o transplante das mudas. O B foi aplicado dissolvido em $10 \mathrm{~mL}$ de água e incorporado em cada vaso no dia anterior ao plantio das mudas, nas doses de B: $0 ; 5 ; 10 ; 20$ e 30 mg.kg-1 de solo em três níveis de $\mathrm{pH}$ do solo: 5,5; 6,2 e 7,3. As fontes de $\mathrm{N}$ testadas foram: sulfato de

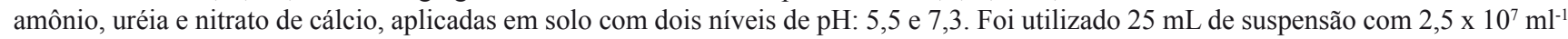
esporos de repouso do patógeno, aplicada no colo de cada planta, no transplante das mudas. Aos 45 dias após a inoculação foram realizadas as avaliações. A severidade da doença foi maior (70\%) com doses de B menores que $10 \mathrm{mg} \cdot \mathrm{kg}^{-1}$ e pH menor que 6,0 . A menor severidade (26\%) foi verificada em pH maior que 6,5 com 10 a $30 \mathrm{mg} \cdot \mathrm{kg}^{-1} \mathrm{de}$ B. A severidade da doença foi menor quando o nitrato de cálcio foi utilizado em solo com pH 6,5 do que em outros tratamentos. Observou-se correlação negativa entre os teores foliares de $\mathrm{N}$, Ca, Mg e B e a severidade da doença. Os resultados obtidos indicaram que o plantio em solo com pH maior que 6,5 associado à aplicação de 10 a 30 mg. $\mathrm{kg}^{-1}$ de boro e o uso do nitrato de cálcio como fonte de adubação nitrogenada apresentam resultados positivos na redução da doença. Palavras-chave: controle cultural, galha, nutrição mineral, Brassica rapa var. pekinensis.
\end{abstract}

\begin{abstract}
Influence of boron, nitrogen sources and soil pH on the control of club root of crucifers caused by Plasmodiophora brassicae Effects of Boron (B) doses and nitrogen sources $(\mathrm{N})$ at different levels of soil $\mathrm{pH}$ on the control of clubroot was investigated in transplants of chinese cabbage grown in pots containing field soil.. B dissolved in water was applied at $0,5,10,20$ and 30 mg.kg-1 of soil of $\mathrm{pH} 5.5,6.2$ and 7.3 on the day before transplanting. N sources tested (ammonium sulfate, urea and calcium nitrate) were applied to soil of $\mathrm{pH} 5.5$ and 7.3. Seedlings were inoculated with a suspension of resting spores of the pathogen at transplanting and assessed for clubroot severity after 45 days. The disease was severe (70\%) when the B dose was less than $10 \mathrm{mg}^{-\mathrm{kg}^{-1}}$ and the $\mathrm{pH}$ less than 6.0 , but milder (26\%) when the B dose was 10 to $30 \mathrm{mg} \cdot \mathrm{kg}^{-1}$ soil at $\mathrm{pH}$ values higher than 6.5 . Clubroot was least severe when calcium nitrate was used in soil of $\mathrm{pH}$ 6.5. Clubroot severity correlated negatively with concentrations of $\mathrm{N}, \mathrm{Ca}, \mathrm{Mg}$ and $\mathrm{B}$ in the foliage. We conclude that use of 10 to 30 mg. $\mathrm{kg}^{-1}$ boron and calcium nitrate in soil of $\mathrm{pH} \mathrm{6,5} \mathrm{or} \mathrm{7,3} \mathrm{was} \mathrm{effective} \mathrm{in} \mathrm{reducing} \mathrm{club} \mathrm{root} \mathrm{severity} \mathrm{.}$
\end{abstract}

Keywords: cultural control, gall, mineral nutrition, Brassica rapa var. pekinensis.

\section{INTRODUÇÃO}

A hérnia das crucíferas (Plasmodiophora brassicae Woron.) tem limitado os plantios de repolho (Brassica oleracea L. var. capitata L.), couve-flor (Brassica oleracea L. var. botrytis L.), brócolis (Brassica oleracea L. var. italica Plenck), couve-chinesa (Brassica pekinensis L.), mostarda (Brassica juncea L. Czern. e Cross) e rúcula (Brassica juncea L. Czern. e Cross), na Região Metropolitana de Curitiba, PR (Ruaro et al., 2003). No Brasil a doença está presente nas regiões Sul e Sudeste, especialmente em locais com clima úmido e temperaturas mais baixas (Maringoni, 1995). No processo de colonização do hospedeiro, o patógeno induz o aparecimento e desenvolvimento de galhas no sistema radicular, o qual sofre apodrecimento e decomposição antecipada culminando com a liberação de esporos de resistência. Nesta condição, o patógeno pode permanecer no solo por longos períodos. A doença é favorecida quando a temperatura oscila entre 18 e $25^{\circ} \mathrm{C}$, o solo é arenoso, e o pH é ácido. Esta última condição é preponderante no desencadeamento e desenvolvimento da infecção (Agrios, 1997).

O controle químico é difícil por se tratar de um patógeno de solo e por existirem poucos fungicidas eficientes, e são poucas as variedades de brássicas resistentes ao patógeno (Dixon \& Robinson, 1996). A recomendação de controle por rotação de culturas com espécies não suscetíveis e principalmente a elevação do $\mathrm{pH}$ com calagem 
são enfatizadas como medidas importantes para reduzir o nível de inóculo no solo. Segundo Webster \& Dixon (1991), a doença é mais severa em $\mathrm{pH}$ menores que 5,7; entre 5,7 e 6,2 a severidade da doença decresce e acima de $\mathrm{pH}$ 7,8 o patógeno é complemente inibido.

A resistência das plantas à doenças, mesmo sendo geneticamente controlada, poderá ser afetada pelos fatores ambientais e a nutrição mineral é um fator que pode ser manipulado visando o controle das doenças. Os nutrientes maximizam a resistência das plantas a determinadas doenças, facilitam a evasão e alteram o ambiente externo, atuando na sobrevivência, germinação e penetração dos patógenos, interferindo assim na interação planta x patógeno $\mathrm{x}$ ambiente. Práticas de controle cultural de doenças como o ajuste do $\mathrm{pH}$ do solo pela calagem pode influenciar a severidade das doenças diretamente ou por meio da interação com os nutrientes, afetando sua solubilidade. Para o caso do boro, este fato pode ser observado principalmente em solos ácidos que tendem a ser deficientes neste elemento e especialmente em solos tropicais onde a deficiência de boro é prevalente, o que pode levar ao aumento da incidência de doenças (Marschner, 1996; Huber \& Thompson, 2007).

O efeito do nitrogênio, fósforo, potássio, cálcio, silício e micronutrientes têm sido mais amplamente relatados para outras doenças (Datnoff et al., 2007). O mesmo não acontece para a hérnia das crucíferas, cuja disponibilidade de trabalhos sobre o boro é escassa (Stangoulios \& Graham, 2007). Trabalhos pioneiros sobre a influência desse elemento no controle de P. brassicae são destacados na revisão organizada por Dixon (1996). Estes trabalhos (Dixon et al., 1987; Dixon \& Webster, 1988; Webster \& Dixon, 1991) relatam redução na severidade e incidência da doença por meio da manipulação do boro e do $\mathrm{pH}$ do solo, destacando seus efeitos antagônicos na patogênese causada por $P$. brassicae, exercidos possivelmente por meio da repressão na morfogênese do patógeno, reduzindo a presença de plasmódios e esporângios diferenciados e aptos a causarem infecções. Entretanto, existe nesses trabalhos grande variação na dose de boro avaliada. A exemplo, Webster \& Dixon (1991) e Nott et al. (1999), que trabalhando com solução nutritiva, avaliaram doses de $\mathrm{B}$ de 1 a $70 \mathrm{mg} \mathrm{kg}^{-1}$. Como existe um limite estreito entre a quantidade de boro requerida e a fitotóxica para a planta, e também uma variação no comportamento deste elemento nos diferentes tipos de solos (Marschner, 1996), há a necessidade de se avaliar a dose de boro e $\mathrm{pH}$ do solo mais adequados para o controle de P. brassicae em solos brasileiros.

O produtor na busca de maiores rendimentos e de um produto final mais atrativo aplica grandes quantidades de fertilizantes nitrogenados. Ao ser adicionada ao solo, cada fonte de $\mathrm{N}$ apresenta comportamento específico, podendo estar nas formas amídica, amoniacal ou nítrica. Quando o processo de nitrificação converte o íon amônio a nitrato, íons hidrogênio são liberados, isto é uma fonte de acidez para o solo (Mengel \& Kirkby, 1978). A intensidade de acidificação depende da fonte do fertilizante utilizado (Tisdale \& Nelson 1975). O pH da rizosfera é reduzido pela absorção radicular de $\mathrm{NH}_{4}$, e aumentado quando $\mathrm{NO}_{3}$ é absorvido (Huber \& Thompson, 2007). Como a hérnia das crucíferas é favorecida pelo $\mathrm{pH}$ baixo, a utilização de fontes de fertilizantes que deixam nos solos resíduos ácidos, provocando o aumento da acidez poderia interferir na eficiência da calagem como medida de controle desta doença.

Pesquisas referentes às fontes de $\mathrm{N}$ no controle de $P$. brassicae também são escassas e conta-se com os trabalhos de Dobson et al. (1983) e de Klasse (1996), que relataram decréscimo na incidência e severidade da doença com o uso do sulfato de amônio. Contudo, Dixon (2009) destaca que fatores como nitrogênio, boro e $\mathrm{pH}$ do solo podem influenciar de modo diferente algumas raças fisiológicas de P. brassicae. Pouco se sabe sobre as populações brasileiras deste patógeno, mas há indícios sobre diferenças existentes entre populações heterogêneas (Cruz et al., 2008). Assim, é fundamental o entendimento dos efeitos desses fatores em condições brasileiras. O objetivo deste trabalho foi determinar o efeito de doses de $\mathrm{B}$, de fontes de $\mathrm{N}$ e do $\mathrm{pH}$ do solo no controle da hérnia das crucíferas (Plasmodiophora brassicae).

\section{MATERIAL E MÉTODOS}

A planta hospedeira utilizada nos experimentos foi couve-chinesa suscetível (Brassica rapa var. pekinensis) Pak choi, cujas mudas foram produzidas em bandejas, contendo substrato inerte à base de vermiculita. Aos 10 dias após a semeadura, as mudas foram transplantadas (uma planta por vaso) nos respectivos tratamentos. Foram utilizados vasos de alumínio com capacidade para $1,5 \mathrm{~kg}$ de solo.

Foram conduzidos dois experimentos em condições de casa-de-vegetação, no Setor de Ciências Agrárias da UFPR, em Curitiba, PR. O solo utilizado nos dois experimentos foi o cambissolo háplico Ta Distrófico típico, com textura argilo-arenosa retirado de área não cultivada, na Fazenda Experimental do Canguiri da UFPR, na profundidade de zero a $20 \mathrm{~cm}$, que foi submetido à análise química no Laboratório de Fertilidade (UFPR), (Tabela 1).

Para o ajuste do $\mathrm{pH}$ do solo a necessidade de calagem foi baseada na curva de calibração, que indicou as doses de 2,4; 4,7 e 9,5 T de calcárioha ${ }^{-1}$, para os valores de $\mathrm{pH}$ de 5,$5 ; 6,2$ e 7,3 respectivamente. Foi utilizado calcário calcítico com PRNT de 104,5\%, aplicado ao solo 30 dias antes do transplante das mudas, para vasos contendo $1,5 \mathrm{~kg}$ de solo. Durante este período os vasos foram irrigados com água destilada e deionizada até a saturação para otimizar a reação do calcário no solo. As características químicas do solo após a aplicação do calcário (antes do plantio) estão apresentadas na Tabela 1. As adubações seguiram as recomendações apresentadas na Comissão de Química e Fertilidade do Solo (1995). 
Influência do boro, de fontes de nitrogênio e do $\mathrm{pH}$ do solo...

TABELA 1 - Características químicas do solo utilizado para avaliação de doses de $\mathrm{B}$, fontes de $\mathrm{N}$ e $\mathrm{pH}$ do solo no controle de $P$. brassicae

\begin{tabular}{|c|c|c|c|c|c|c|c|c|c|c|c|}
\hline \multirow{2}{*}{$\begin{array}{c}\text { Doses de Calcário } \\
\text { T.ha }^{-1}\end{array}$} & \multirow{2}{*}{$\underset{\mathrm{CaCl}_{2}}{\mathrm{pH}}$} & $\mathrm{AH}^{3}$ & $\mathbf{H}+\mathbf{A l}$ & $\mathrm{Ca}^{+2}+\mathrm{Mg}^{+2}$ & $\mathrm{Ca}^{+2}$ & $\mathbf{K}^{+}$ & $T$ & \multirow{2}{*}{$\underset{\text { mg.dm }}{P}$} & \multirow{2}{*}{$\underset{\text { mg.kg }}{\text { B }}$} & \multirow{2}{*}{$\begin{array}{c}\mathrm{C} \\
\text { g.dm }\end{array}$} & \multirow{2}{*}{$\begin{array}{l}\mathrm{V} \\
\%\end{array}$} \\
\hline & & \multicolumn{6}{|c|}{ cmolc. $_{c} \mathrm{dm}^{-3}$} & & & & \\
\hline 0 & 4,4 & 3,5 & 12,10 & 2,80 & 1,70 & 0,28 & 15,2 & 1,0 & 0,5 & 8,8 & 20 \\
\hline 2,4 & 5,5 & 0,0 & 2,70 & 14,20 & 9,60 & 0,20 & 17,1 & 1,2 & 0,7 & 9,4 & 84 \\
\hline 4,7 & 6,2 & 0,0 & 1,20 & 15,00 & 9,80 & 0,16 & 17,0 & 1,0 & 0,6 & 9,8 & 86 \\
\hline 9,5 & 7,3 & 0,0 & 1,00 & 14,70 & 10,20 & 0,15 & 17,8 & 0,7 & 0,4 & 11,4 & 87 \\
\hline
\end{tabular}

\section{Influência do boro e do pH do solo no controle de $P$. brassicae}

As doses de boro utilizadas foram: $0 ; 5 ; 10 ; 20$ e 30 $\mathrm{mg} \mathrm{kg}^{-1}$ de solo, o equivalente a: $0 ; 75 ; 150 ; 300$ e $450 \mathrm{mg}$ de Bórax $/ 1,5 \mathrm{~kg}$ de solo, dissolvidas em $10 \mathrm{~mL}$ de água e incorporadas em cada vaso individualmente, no dia anterior ao plantio das mudas. As quantidades aplicadas por vaso foram: $150 \mathrm{mg}$ de $\mathrm{N}, 375 \mathrm{mg}$ de $\mathrm{P}_{2} \mathrm{O}_{5}$ e $225 \mathrm{mg}$ de $\mathrm{K}_{2} \mathrm{O}$. As fontes utilizadas foram respectivamente: nitrato de cálcio, superfosfato simples e cloreto de potássio. Em cobertura, foram aplicadas $45 \mathrm{mg}$ de N/1,5 $\mathrm{kg}$ de solo na forma de nitrato de cálcio, aos 15 e 30 dias após o transplante.

\section{Influência de fontes de nitrogênio e do $\mathrm{pH}$ do solo no controle de $P$. brassicae}

Os valores de $\mathrm{pH}$ utilizados foram 5,5 e 7,3. As fontes de nitrogênio utilizadas nos tratamentos foram a uréia - $\mathrm{CO}\left(\mathrm{NH}_{2}\right)_{2}$ (44\% de nitrogênio), o sulfato de amônio $\left(\mathrm{NH}_{4}\right)_{2} \mathrm{SO}_{4}(20 \%$ de $\mathrm{N})$ e o nitrato de cálcio $\mathrm{CaNO}_{3}(15 \%$ de $\mathrm{N})$. Foram aplicados na base, $100 \mathrm{~kg}$ de $\mathrm{N} \cdot \mathrm{ha}^{-1}$ o equivalente a $150 \mathrm{mg}$ de $\mathrm{N} / 1,5 \mathrm{~kg}$ de solo. Em cobertura foram utilizados $90 \mathrm{~kg}$ de $\mathrm{N} \cdot \mathrm{ha}^{-1}$ ou $135 \mathrm{mg}$ de $\mathrm{N} \cdot 1,5 \mathrm{~kg}^{-1}$ aplicados em duas vezes, aos 15 e aos 30 dias após o transplante. As quantidades de fósforo, de potássio e de boro aplicadas por vaso foram: $375 \mathrm{mg}$ de $\mathrm{P}_{2} \mathrm{O}_{5}$ e $225 \mathrm{mg}$ de $\mathrm{K}_{2} \mathrm{O}$ e $5,3 \mathrm{mg}$ de $\mathrm{B}$. As fontes utilizadas foram respectivamente: superfosfato simples, cloreto de potássio e bórax. No tratamento sem aplicação de nitrogênio, as plantas serviram como padrão.

Para a produção do inóculo para cada experimento foram utilizadas raízes de couve- chinesa com galhas, coletadas de plantas com 50 dias e naturalmente infectadas, em plantio comercial da cultura, localizado no município de Colombo PR. A coleta foi realizada após inspeção visual, selecionando-se aquelas plantas que apresentavam sintoma reflexo de murcha da parte aérea. Entre estas plantas, escolheram-se as que apresentavam galhas bem desenvolvidas, descartando-se aquelas em início de decomposição. As galhas foram lavadas e trituradas em liquidificador por 60 segundos, na proporção de $135 \mathrm{~g}$ de galhas para $100 \mathrm{~mL}$ de água esterilizada. A extração dos esporos de repouso foi realizada conforme metodologia proposta por Naiki \& Dixon (1987). A inoculação foi realizada utilizando-se $25 \mathrm{~mL}$ de suspensão com $2,5 \times 10^{7}$ $\mathrm{mL}^{-1}$ esporos de repouso do patógeno, aplicada no colo de cada planta, logo após o transplante das mudas. O solo encontrava-se com umidade à capacidade de campo, e após a aplicação foi realizada rega com $75 \mathrm{~mL}$ de água aplicada em cada vaso, com o objetivo de distribuir o inóculo no solo.

Aos 45 dias após a inoculação foram realizadas as avaliações. A severidade da doença expressa em porcentagem foi determinada através da estimativa da área radicular doente em relação à área radicular sadia (Dixon \& Robinson, 1996). A parte aérea das plantas foi pesada e submetida à secagem em estufa de circulação forçada a $70^{\circ} \mathrm{C}$ por 72 horas, para determinação da fitomassa da matéria fresca e seca. Foi realizada também a coleta de amostra para a determinação da concentração de N, P, K, Ca, Mg e B no tecido foliar. Cada amostra foi constituída pela nervura central de 8 folhas externas obtidas de quatro plantas de cada unidade experimental (van Raij, 1991).

Os experimentos foram conduzidos em blocos ao acaso, e tratamentos em ensaio fatorial. Cada tratamento contou com quatro repetições, onde cada repetição foi composta de um vaso com uma planta. Os resultados foram submetidos à análise de variância e, quando significativos à análise de regressão polinomial para o experimento 1 e as médias comparadas pelo teste de Tukey a $5 \%$ de probabilidade para o experimento 2 . O logaritmo das variáveis massa foliar fresca (MFF) e massa foliar seca (MFS) foram sugeridos como transformação para aproximação da distribuição normal dos erros.

\section{RESULTADOS E DISCUSSÃO}

\section{Influência do boro e do pH do solo no controle de Plasmodiophora brassicae}

A variação nas doses de boro e nos níveis de $\mathrm{pH}$ do solo, e as combinações entre estes fatores influenciaram significativamente a severidade da doença. Observa-se os maiores índices de severidade $(>70 \%)$ para interação $\mathrm{pH}$ mais ácido $(<6,0)$ e baixos níveis de $\mathrm{B}$ no solo, menos que $10 \mathrm{mg} \mathrm{kg}^{-1}$ de B (Figura 1A). Baixo índice de severidade, em torno de $26 \%$, quando o pH do solo foi 
$z=81.52+5.444^{*} x+0.086^{*} y-1.216^{*} x^{*} x-0.333^{*} x^{*} y+0.034^{*} y^{*} y$ $\mathrm{R}^{2}=-0,57$

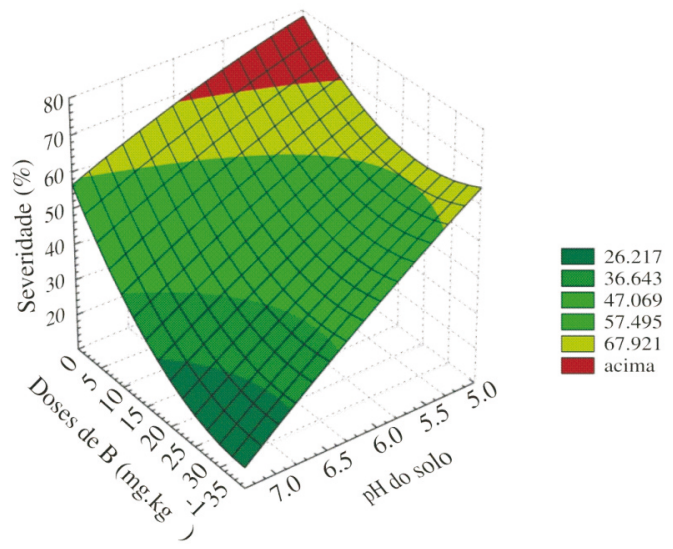

C

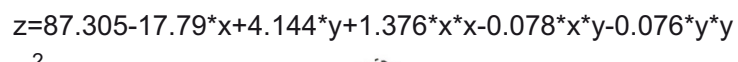
$R^{2}=0,91$
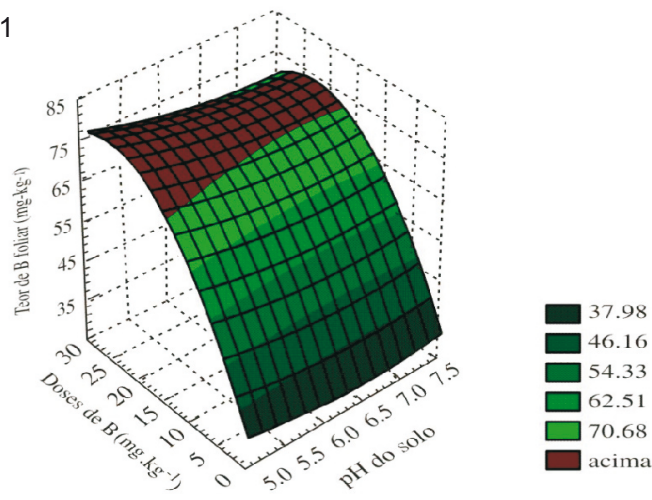

E

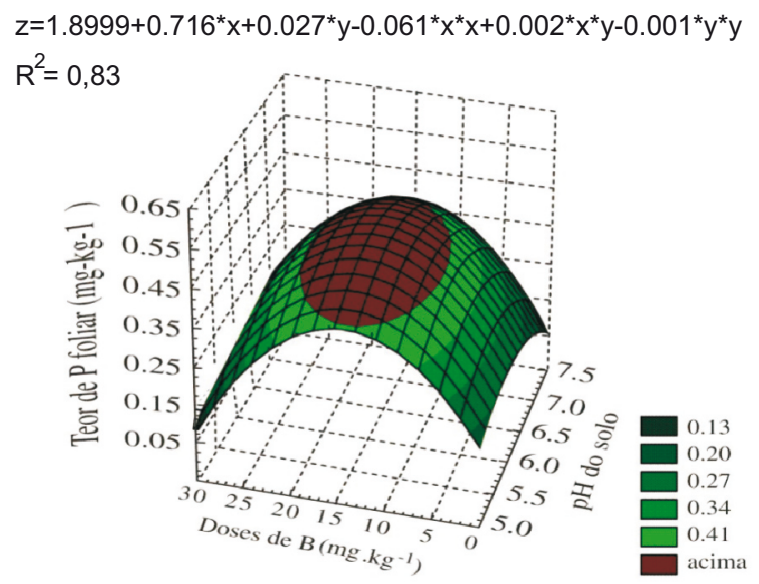

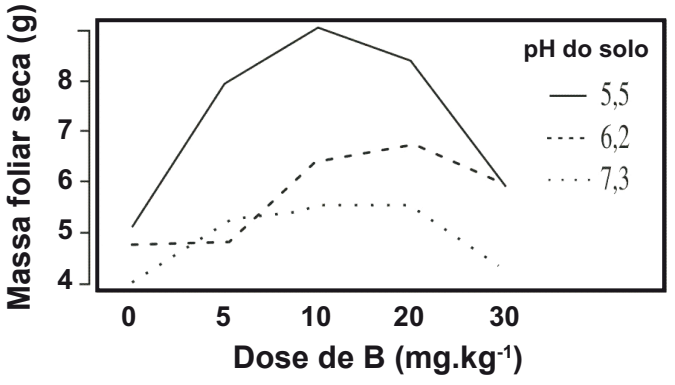

D

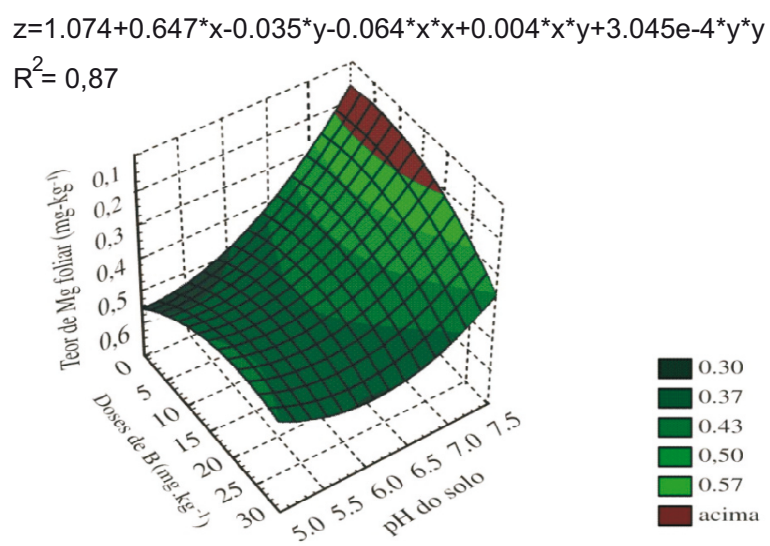

$\mathbf{F}$

$z=23.109+8.35^{*} x+0.235^{*} y-0.657^{*} x^{*} x-0.013^{*} x^{*}-0.005^{*} y^{*} y$

$R^{2}=-0,63$

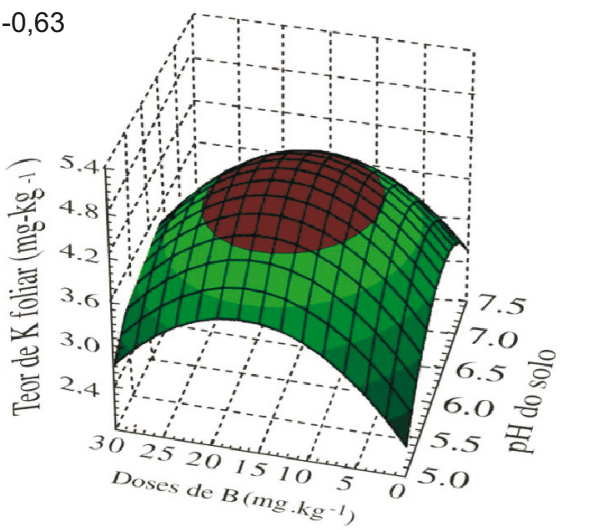

FIGURA 1 - A. Superfície polinomial ajustada para a severidade da hérnia das crucíferas; C. para os teores foliares de boro; D. magnésio; E. fósforo; F. potássio; e B. a interação entre níveis de $\mathrm{pH}$ do solo x doses de boro na produção de massa foliar seca de plantas de couve chinesa infectadas por $P$. brassicae, em relação às doses de $\mathrm{B}$ e três níveis de $\mathrm{pH}$ do solo. 
maior que 6,5 na faixa de 10 a $30 \mathrm{mg} \cdot \mathrm{kg}^{-1}$ de $\mathrm{B}$ no solo. Sendo que à medida que se elevou o $\mathrm{pH}$ do solo de 6,2 para 7,3 e a quantidade de B de 20 para $30 \mathrm{mg} \mathrm{kg}^{-1}$ houve significativa redução da severidade com valores próximos de $20 \%$. Webster \& Dixon (1991), trabalhando com solução nutritiva, verificaram consideráveis reduções na severidade da doença em couve-chinesa, com $10 \mathrm{mgkg}^{-1}$ de B e pH 7,2 , dose essa abaixo daquela que proporcionou menor severidade no presente trabalho. A diferença nos resultados obtidos pode ser devida ao uso de solução nutritiva pelos autores e de solo no presente trabalho. Segundo Marschner (1996), o boro apresenta variação no seu comportamento nos solos. As concentrações ou atividades das formas iônicas deste elemento na solução do solo, que são usadas pelas plantas, são bastante dependentes do $\mathrm{pH}$, e sua disponibilidade é maior em $\mathrm{pH}$ menor que 6,0. Se o pH aumenta demais, como conseqüência de calagem excessiva, por exemplo, o B entra em combinação com as argilas e com a própria matéria orgânica resultando em compostos de menor disponibilidade. Quanto ao mecanismo exercido pelo boro no controle da doença, Dixon (1996) descreve que existe relação entre a severidade da doença e a presença de plasmódios e esporângios diferenciados e aptos a causarem infecções, quando da manipulação do boro e do $\mathrm{pH}$ do solo.

Houve diferença significativa entre os níveis de $\mathrm{pH}$ e as doses de boro adicionadas ao solo, assim como interação entre estes sobre a produção de massa foliar seca das plantas (Figura 1-B). A maior massa foliar foi obtida com doses de $B$ entre 10 e $20 \mathrm{mg}^{-1} \mathrm{~kg}^{-1}$, com destaque para $10 \mathrm{mg} \mathrm{kg}^{-1}$ de $\mathrm{B}$ entre os valores de $\mathrm{pH}$ de 6,2 e 7,3. Estes resultados evidenciam a importância da aplicação deste elemento, principalmente quando se utiliza a calagem para corrigir o pH do solo para níveis mais elevados.

Foi observada correlação entre os teores de $\mathrm{N}, \mathrm{Ca}$ e B na parte aérea das plantas e a severidade da doença. A correlação entre a dosagem aplicada desses elementos ao vaso e a produção de massa foliar seca foi positiva (Tabela 2), indicando que quanto maior o teor de $\mathrm{N}, \mathrm{Ca}$ e $\mathrm{B}$ apresentado pelas plantas, menor foi a severidade da doença e maior a produção de massa foliar seca. Segundo Marschner (1996), o conteúdo de cálcio nos tecidos das plantas afeta a incidência de doenças parasíticas por ser essencial para a estabilidade das biomembranas, onde poligalacturonatos de cálcio são requeridos na lamela média para que haja estabilidade da parede celular. Dixon \& Webster (1988) e Dixon et al. (1987) afirmam que o cálcio pode inibir o amadurecimento dos esporângios de $P$. brassicae e seu modo de ação pode ser independente ou associado com os efeitos do $\mathrm{B}$ e do $\mathrm{pH}$ do solo na redução da severidade da doença. A interação entre B e Ca poderia explicar o efeito deste elemento na redução da severidade e no aumento da produção de massa foliar seca observada no presente trabalho.

A quantidade de B na parte aérea das plantas foi maior com uso de doses elevadas deste elemento aplicadas ao solo, sendo que com $\mathrm{pH}$ 7,3 verificou-se menor absorção que nos demais níveis de pH (Figura 1-C). Enquanto que as quantidades de $\mathrm{N}, \mathrm{Ca}$ e $\mathrm{Mg}$ (Figura 1-D) foram maiores em $\mathrm{pH}$ 7,3. Para $\mathrm{P}$ e K a quantidade foi mais elevada nos tratamentos com aplicação de $20 \mathrm{mgkg}^{-1}$ de B e pH 6,2, com valores decrescendo a medida que se elevou o $\mathrm{pH}$ para 7,3 (Figura 1 E-F). O comportamento observado na absorção dos nutrientes pode ser atribuído ao efeito indireto da calagem, que estabelece uma relação entre o pH e a disponibilidade de elementos. A disponibilidade dos íons de $\mathrm{Ca}$ e $\mathrm{Mg}$ na solução do solo ou no complexo de troca é aumentada com a elevação do $\mathrm{pH}$ do solo, e a disponibilidade de $\mathrm{P}$ é diminuída em valores baixos de $\mathrm{pH}$, ou quando o $\mathrm{pH}$ é maior que 7,0 (Tisdale \& Nelson, 1975).

\section{Influência de fontes de nitrogênio e do $\mathrm{pH}$ do solo no controle de Plasmodiophora brassicae}

Houve diferença significativa entre as fontes de nitrogênio e os níveis de $\mathrm{pH}$ do solo, bem como a interação entre estes fatores sobre a severidade da doença, que foi menor, em torno de $35 \%$, utilizando-se o nitrato de cálcio ou a uréia como fonte de nitrogênio, em $\mathrm{pH} 7,3$. No tratamento sem aplicação de nitrogênio e pH mais ácido $(5,5)$ a severidade da doença atingiu níveis superiores a 75\% (Figura 2-A), e nos tratamentos com a aplicação de sulfato de amônio a severidade também foi elevada, possivelmente devido ao maior efeito acidificante desta fonte de $\mathrm{N}$. Os valores de $\mathrm{pH}$ que inicialmente eram de 5,5 e 7,3, ao final do experimento foram, respectivamente: 5,1 e 6,6 com aplicação de sulfato de amônio, 5,2 e 6,8 com aplicação de uréia e 5,5 e 7,1 com aplicação de nitrato de cálcio. Essa redução do $\mathrm{pH}$ apresentada pelas fontes utilizadas se deve ao fato de que o sulfato de amônio é fonte de $\mathrm{N}$ amoniacal $\left(\mathrm{NH}_{4}^{+}\right)$, que quando adicionado ao solo o íon $\mathrm{NH}_{4}^{+}$produz $\mathrm{H}^{+}$promovendo a acidez (van Raij, 1991), no presente trabalho, esta foi a fonte que teve efeito mais pronunciado na redução do $\mathrm{pH}$. A uréia com $\mathrm{N}$ na forma amídica, não contém $\mathrm{NH}_{4}^{+}$, mas no solo pode ser hidrolisada rapidamente, e produzir íons de amônio e bicarbonato. Uma vez convertida em amônio, a uréia comporta-se como qualquer outro fertilizante nitrogenado amoniacal. E devido à produção final de $\mathrm{H}^{+}$a adição de uréia também pode promover o abaixamento do $\mathrm{pH}$ do solo. Enquanto que o nitrato de cálcio é um fertilizante que contém $\mathrm{N}$ na forma nítrica $\left(\mathrm{NO}_{3}\right)$ e sua aplicação não leva à redução do pH do solo (Malavolta, 1981). A redução nos valores de $\mathrm{pH}$ observada no presente trabalho pode ser representativa para o caso da $P$. brassicae, pois conforme citado anteriormente, a doença é severa até pH 5,7; decrescendo entre 5,7 e 6,2, e acima de 7,2 o patógeno pode ser completamente inibido.

Além do efeito direto das fontes sobre o $\mathrm{pH}$ do solo, segundo Huber \& Thompson (2007), a forma de nitrogênio (amônio ou nitrato) disponível ao hospedeiro ou ao patógeno afeta a severidade ou resistência às doenças. Dobson et al. (1983) também verificaram em plantas de couve chinesa que a incidência da doença foi maior com o uso do sulfato de amônio, com ou sem calcário, e o nitrato de cálcio reduziu a severidade, por ser capaz de aumentar o $\mathrm{pH}$ do solo e da 
A

C
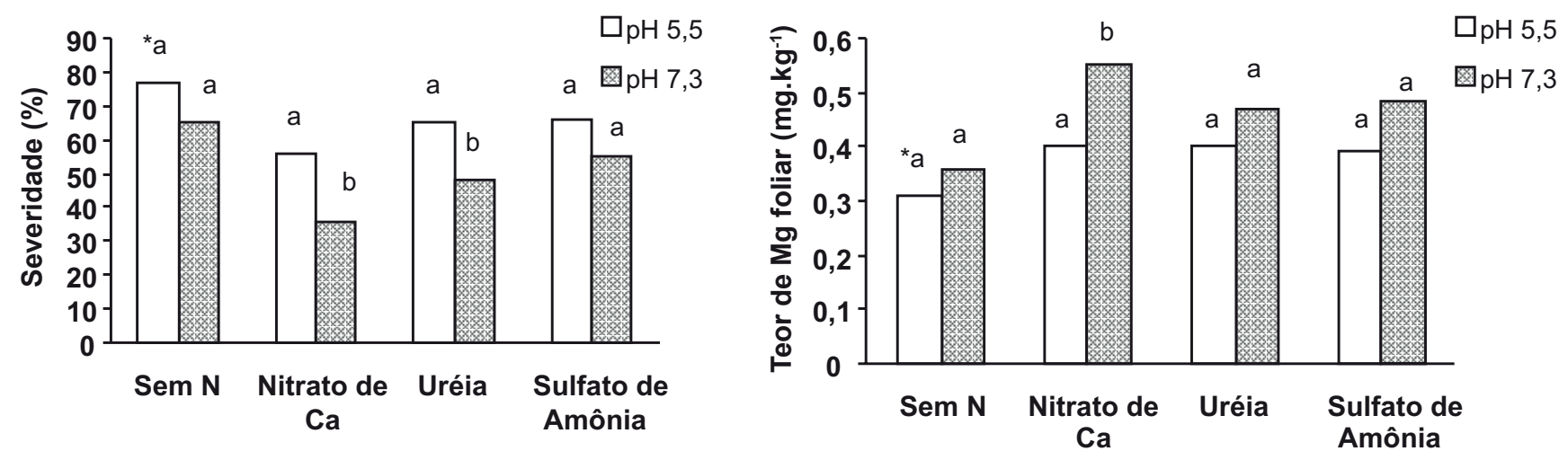

B

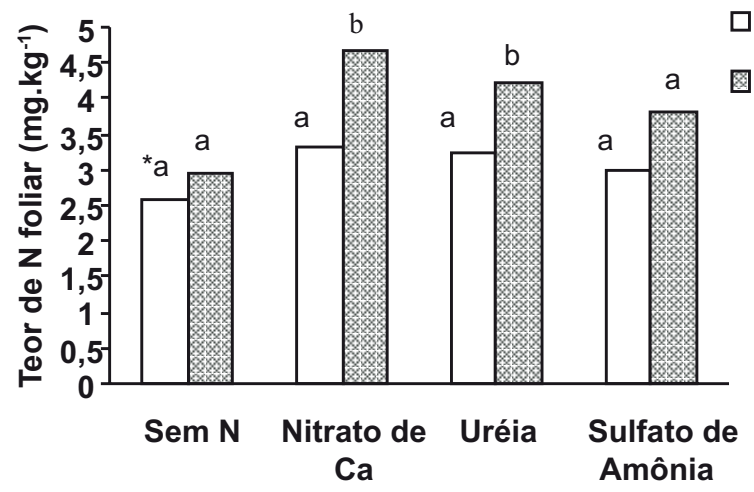

$\square \mathrm{pH} 5,5$

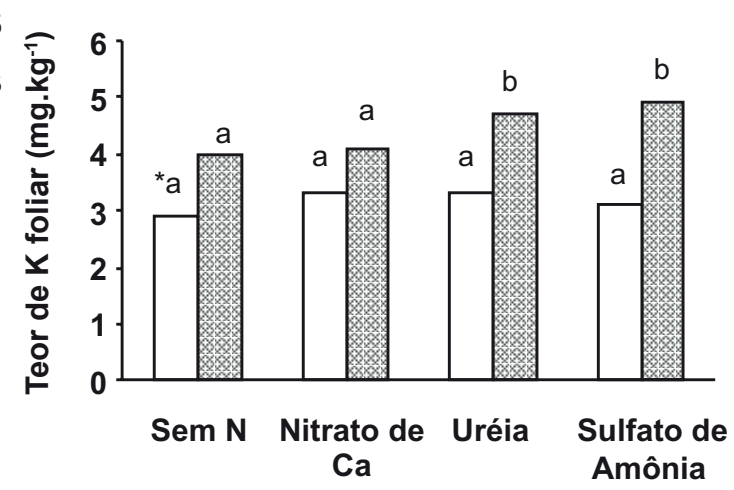

$\square \mathrm{pH} 5,5$

圈 $\mathrm{pH} 7,3$

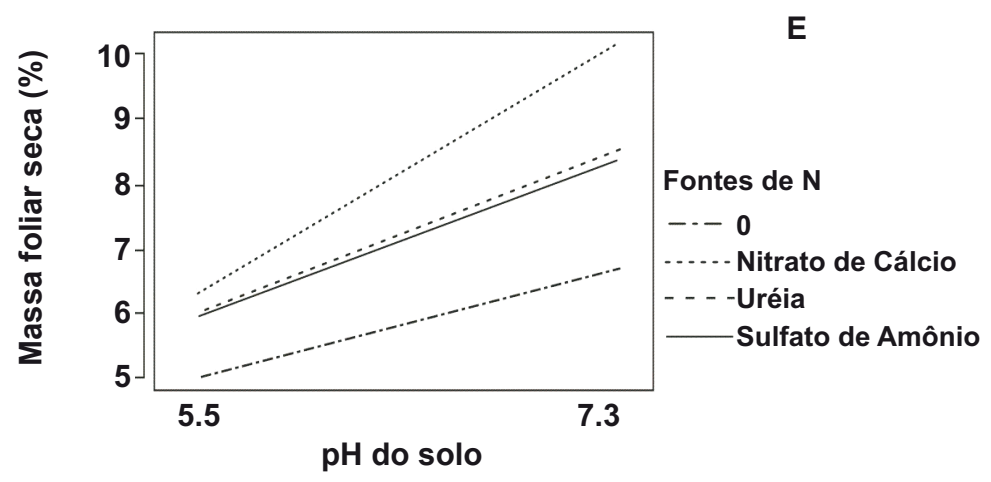

FIGURA 2 - A. Efeito do pH do solo e de fontes de N, na severidade da doença; B. nos teores foliares de nitrogênio; C. magnésio; D. potássio; e E. na produção de massa foliar fresca de plantas de couve chinesa infectadas por P. brasicae. *Médias seguidas de mesma letra não diferem entre si pelo teste de Tukey $(\mathrm{P}<0,05)$.

rizosfera. A diferença nos valores de $\mathrm{pH}$ observada entre as fontes neste trabalho confirma resultados observados por Carvalho et al. (2005) que, ao avaliarem o efeito da fonte de nitrogênio sobre o início do processo de infecção de raízes de mudas de tomateiro por F. oxysporum f. sp. Lycopersici, verificaram que o N-NO ${ }_{3}$ proporcionou maiores valores de pH e reduziu a taxa de adesão de conídios e da colonização

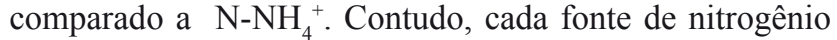
pode aumentar ou diminuir a severidade, dependendo do patossistema em questão (Huber \& Thompson, 2007). Por exemplo, Rodrigues et al. (2002) verificaram que o uso do nitrogênio na forma nítrica contribuiu mais para o aumento da podridão-radicular causado pela Rhizoctonia em feijoeiro, que a fonte amoniacal. 
Influência do boro, de fontes de nitrogênio e do $\mathrm{pH}$ do solo...

TABELA 2 - Coeficientes de correlação entre variáveis medidas em plantas de couve chinesa infectadas por $P$. brassicae e submetidas a cinco doses de $\mathrm{B}$ em três níveis de $\mathrm{pH}$ do solo e a três fontes de $\mathrm{N}$ em dois níveis de $\mathrm{pH}$ do solo

\begin{tabular}{llclc}
\hline \hline Ensaio & Variável & N & Ca & B \\
\hline Doses de B x pH do solo & Massa foliar seca & $0,78^{*}$ & $0,85^{*}$ & $0,30^{*}$ \\
& Severidade & $0,52^{*}$ & $0,63^{*}$ & $0,56^{*}$ \\
& Massa foliar seca & $0,83^{*}$ & $0,60^{*}$ & $0,81^{*}$ \\
Fontes de N x pH do solo & Severidade & $0,92^{*}$ & $0,52^{*}$ & $0,82^{*}$ \\
\hline
\end{tabular}

*Correlações significativas em nível de $5 \%$ de probabilidade.

Houve efeito significativo entre os fatores fontes de $\mathrm{N}$ e pH do solo e sua interação no conteúdo de $\mathrm{N}, \mathrm{Ca}$ e B na parte aérea das plantas. A correlação foi negativa entre a quantidade desses elementos e a severidade da doença, e positiva para a massa foliar seca (Tabela 2). Para o $\mathrm{N}$ o coeficiente de correlação foi de $-0,92$, à medida que aumentou a quantidade de nitrogênio houve redução na severidade da doença. A maior quantidade de nitrogênio na parte aérea das plantas foi verificada quando se utilizou o nitrato de cálcio ou a uréia, combinados com pH 7,3 (Figura 2-B). A adição de fertilizante na forma nítrica manteve o $\mathrm{pH}$ maior que 7,0 até o final do experimento, fato que pode ter contribuído para reduzir a severidade da doença e aumentar a produção de massa foliar seca nesses tratamentos (Figura 2-E). As elevadas quantidades de nitrogênio contidas nas brássicas indicam a necessidade alta do nutriente para estas plantas e a calagem tem um efeito pronunciado no aproveitamento do nitrogênio e, assim, influi nas respostas ao nutriente aplicado em adubações (Castellane et al., 1993), visto que foi no maior valor de $\mathrm{pH}$ do solo, que houve maior resposta à adubação nitrogenada, em termos de crescimento da planta.

A importância do Ca na redução da severidade de galhas foi destacada nos resultados do experimento 1. Vale ressaltar que incrementos na concentração deste elemento e aumento do $\mathrm{pH}$ podem ativar mecanismos de inibição a P. brassicae e elevar a resistência do hospedeiro (Webster \& Dixon, 1991). Os teores de cálcio e de magnésio (Figura 2-C) foram superiores nos tratamentos com nitrato de cálcio em $\mathrm{pH}$ 7,3. O Ca e o Mg estão constantemente associados, pois são aplicados ao solo através da calagem (Van Raij, 1991). Além disso, o nitrato de cálcio é um fertilizante que contém $25 \mathrm{~g} \mathrm{~kg}^{-1}$ de Ca e quando solubilizado fornece cálcio para a solução do solo, que é absorvido pelas raízes na forma iônica $\mathrm{Ca}^{++}$(Malavolta, 1981), explicando assim o maior teor deste elemento, nos tratamentos com nitrato de cálcio e $\mathrm{pH}$ 7,3. Foi verificado maior teor de potássio nos tratamentos com pH 7,3 e aplicação de uréia ou sulfato de amônio (Figura 2-D). Os menores teores de K observados nos tratamentos com aplicação de nitrato de cálcio podem estar relacionados à maior disponibilidade de Ca no solo nos tratamentos com esta fonte. Mengel \& Kirkby (1978) descrevem que o efeito provocado por altas concentrações de Ca na absorção de K pelas raízes é um exemplo clássico de inibição competitiva, que ocorre quando dois elementos combinam-se, com o mesmo carregador. Os resultados obtidos no presente trabalho indicaram que o plantio em solo com pH maior que 6,5, associado à aplicação de $10 \mathrm{a}$ $30 \mathrm{mg} \cdot \mathrm{kg}^{-1}$ de boro e o uso do nitrato de cálcio como fonte de adubação nitrogenada, apresentam resultados positivos na redução da doença.

\section{REFERÊNCIAS BIBLIOGRÁFICAS}

Agrios GN (2005) Plant Pathology. $5^{\text {th }}$ Ed. Amsterdam. Elsevier Academic Press.

Carvalho AO, Jacob Neto J, Carmo MGF (2005) Colonização de raízes de tomateiro por Fusarium oxysporum f.sp. lycopersici em solução nutritiva com três fontes de nitrogênio. Fitopatologia Brasileira 30:26-32.

Castellane PD, Cruz MCP, Ferreira ME (1993) Nutrição e adubação de hortaliças. Jaboticabal SP. Associação Brasileira para Pesquisa da Potassa e do Fosfato.

Comissão de Química e Fertilidade do Solo (1995) Recomendações de adubação e de calagem para os estados do Rio Grande do Sul e Santa Catarina. $3^{\text {a }}$ Ed. Passo Fundo RS. Sociedade Brasileira de Ciência do Solo.

Cruz JCS, Souza NL, Nakatani AK, Rosa, DD, Basseto MA, Padovani, CR, Furtado, EL (2008) Caracterização patogênica e molecular de Plasmodiophora brassicae. Tropical Plant Pathology 33:415-424.

Datnoff L, Elmer WH, Huber DM (Eds.) (2007) Mineral Nutrition and Plant Disease. Saint Paul MN. APS Press.

Dixon, GR (1996) Repression of the morphogenesis of Plasmodiophora brassicae Wor. by boron - a review. Acta Horticulturae 407:393-401.

Dixon GR (2009) Plasmodiophora brassicae in its Environment. Journal of Plant Growth Regulation. DOI: 10.1007/s00344-0099098-3. Disponível em: http://www.springerlink.com/content/ wk7415m6u1018082/. Acesso em: 13 jun. 2009.

Dixon GR, Naiki T, Webster MA, Wilson F (1987) Integrated use of boron, calcium cyanamide and nitrogen for control of clubroot (Plasmodiophora brassicae). Proceedings Crop Protection in Northern Britain, pp. 399-404. 
Dixon GR, Robinson DL (1996) The susceptibility of Brassica oleracea cultivars to Plasmodiophora brassicae (clubroot). Plant Pathology 35:101-107.

Dixon GR, Webster MA (1988) Antagonistic effects of boron, calcium and $\mathrm{pH}$ on pathogenesis caused by Plasmodiophora brassicae Woronin (Clubroot), a review of recent work. Crop Research 28: 84-95.

Dobson RL, Gabrielson RL, Baker AS, Bennett L (1983) Effects of lime particle size and distribution and fertilizer formulation on clubroot disease caused by Plasmodiophora brassicae. Plant Disease 67:50-52.

Huber DM, Thompson LA (2007) Nitrogen and plant disease. In: Datnoff LE, Elmer WH, Huber DM (Eds.) Mineral nutrition and plant disease. Saint Paul MN. APS Press. pp. 31-44.

Klasse HJ 1996 Calcium cyanamide - An effective tool to control clubroot, a review. Acta Horticulturae 407:403-410.

Malavolta E (1981) Manual de Química Agrícola Adubos e Adubação. $3^{\text {a }}$. Ed. São Paulo SP. Ceres.

Maringoni AC (1995) Doenças das crucíferas. In: Bergamin Filho A, Kimati H, Amorim L. (Eds.) Manual de Fitopatologia. Vol. 1. Princípios e Conceitos. 3a . Ed. São Paulo SP. Ceres. pp. 318-319.

Marschner H (1996) Relationship between mineral nutrition and plant disease and pests. In: Marschner H (Ed.) Mineral nutrition of higher plants. London. Academic Press. pp. 369-390.
Mengel K, Kirkby EA (1978) Principles of Plant Nutrition. Bern. International Potash Institute.

Naiki T, Dixon GR (1987) The effects of chemicals on developmental stages of Plasmodiophora brassicae. Plant Pathology 36:316-327.

Nott H, Falloon R, Cheah LH (1999) Clubroot control from safe chemicals. Commercial Grower. Purdue University. Dan Egel.

Rodrigues FA, Carvalho EM, Vale RFX (2002) Severidade da podridão-radicular de Rhizoctonia do feijoeiro influenciada pela calagem, e pelas fontes e doses de nitrogênio. Pesquisa Agropecuária Brasileira 37:1247-1252.

Ruaro LR, Lima Neto VC, Nowacki JC (2003) Controle da hérnia das crucíferas na Região Metropolitana de Curitiba. Relatório Técnico. Curitiba PR. Universidade Federal do Paraná. SEAB PR.

Stangoulis JCR, Graham RD (2007). Boron and Plant Disease. In: Datnoff LE, Elmer WH, Huber DM (Eds.) Mineral Nutrition and Plant Disease. Wisconsin. Saint Paul MN. APS Press. pp. 207-214.

Tisdale SL, Nelson WL (1975) Soil fertility and fertilizers. $3^{\text {a }}$. Ed. New York NY. Collier MacMillan International Editions.

Webster MA, Dixon GR (1991) Boron, pH and inoculum concentration influencing colonization by Plasmodiophora brassicae. Mycological Research 95:74-79.

van Raij BV (1991) Fertilidade do Solo e Adubação. Piracicaba SP. Ceres. 